\title{
An eHealth-based intervention against metabolic risks during pregnancy: learnings and challenges
}

\section{Xiaochun Wang}

New century women's and children's hospital

\section{Nan Huang}

Philips Research

Qiao Hua

Philips Research

\section{Dongtao Zhang}

new century women's and children's hospital

Guanghui Li ( $\square$ liguanghui2007@163.com )

new century women's and children's hospital

\section{Ling Fan}

New century women's and children's hospital

\section{Gui Shi}

new century women's and children's hospital

Jun Liu

new century women's and children's hospital

\section{Yafang Jin}

Philips Research

\section{Hehe Ma}

Philips Research

\section{Yana Yuan}

Philips Research

\section{Xuefeng Zhong}

Philips Research

\section{Research article}

Keywords: Connected antenatal care solution, eGWG, GDM

Posted Date: August 18th, 2020

DOI: https://doi.org/10.21203/rs.3.rs-58896/v1 
License: (c) (i) This work is licensed under a Creative Commons Attribution 4.0 International License. Read Full License 


\section{Abstract \\ Background}

Excessive gestational weight gain (eGWG) is one of the major metabolic risks for fetal macrosomia, maternal overweight, gestational diabetes mellitus (GDM), and gestational hypertension during pregnancy, which also increase the risk of postpartum weight retention. Gestational weight gain (GWG) management is rising to be attractive during prenatal care, however, there is still lack of effective tools to assist health care providers (HCPs) to efficiently and continuously implement intervention and monitor from the early phase of pregnancy. The current study tested a prototype eHealth solution aiming to assist clinicians to prevent eGWG and associated gestational metabolic complications.

\section{Methods}

A randomized controlled study was conducted in a private hospital in Beijing, China, with control group $(N=181)$ and intervention group $(N=181)$ recruited in rolling fashion. The control group received standard prenatal care, while the intervention group was additionally provided with the integrated prenatal care eHealth solution, Pregnancy Butler, which includes mobile application-enabled on-line service and off-line consultation session. The prevalence of eGWG, associated metabolic health parameters, and final pregnancy outcome were evaluated.

\section{Results}

Although mostly with normal pre-pregnancy BMI, the incidence for eGWG was high among the study subjects, at $35 \%$. Engagement with the eHealth platform of the intervention group was stable after initial phase, but less than desired. Pregnancy Butler intervention showed significant benefit ( $p$-value $=0.0496)$ in preventing eGWG among obese and overweight subjects. And highly engaged active users in the intervention group had more favorable GWG classification ( $p$-value $=0.022$ ). The time-course data collected indicated that the metabolic risks were largely set early in the first trimester already.

\section{Conclusions}

Our preliminary results indicated that the effectiveness of the eHealth intervention considerably depend on each participant's engagement level and baseline risk factors. In order to achieve more consistent and impactful outcome improvement, focusing on those with pre-existing metabolic risks, increasing engagement and adherence level, and emphasizing intervention from the first trimester could be promising directions.

\section{Trial registration:}


This study was retrospectively registered as "Pregnancy Butler for gestational weight gain management" (ChiCTR1800014647) under the China Clinical Trial Registry in 2018-Jan-26.

http://www.chictr.org.cn/showprojen.aspx?proj=24171

\section{Background}

Excessive gestational weight gain (eGWG) is defined as inappropriately large weight gain during pregnancy assessed according to the guidelines of the Institute of Medicine (IOM). As a risk factor itself, eGWG entails increased chance of gestational diabetes mellitus (GDM), fetal macrosomia, preterm birth, and postpartum weight retention $(1,2)$. At the same time, offsprings of mothers with eGWG are more susceptible to development issue, childhood obesity, and even later-life health conditions such as cardiovascular diseases (3). From mechanistically perspective, maternal eGWG is often accompanied with increase circulating inflammatory cytokines, glucose, insulin, and growth hormones (4). Despite all the above mentioned evidence, there has been an increasing incidence of eGWG in China, with reported incidence as high as $35 \%$ despite their being a population with relatively moderate overweight or obese rate (5).

Many factors have been found to contribute to the development of eGWG. For example, age, ethnicity, higher pre-pregnancy BMI, height, social-economic status, and higher number of prior-parity were known preexisting risks for eGWG $(6,7)$. On the other hand, diet and physical activities are modifiable factors correlate with eGWG risk during pregnancy $(8,9)$. Such modifiable factors thus can serve as tentative target for intervention.

Lifestyle interventions have been explored for eGWG. Small scale intensive-counseling and meal replacement trial significantly lowered gestational weight gain (GWG) among overweight or normal weight women $(10,11)$. Luo et al. also proved the benefit of an intensive prenatal care program including prescribed diet could significantly lower weight gain profile through the pregnancy period, and resulted in fewer GDM, preeclampsia, and macrosomia (12). However, such programs are highly resource demanding and could face application challenge before being widely adopted, with their benefit largely relied on the target population and intensity. A number of studies on lifestyle interventions, summarized by a Cochrane review, suggested that preventing against GDM had moderate to none-effect, citing adherence and baseline condition as the main factors for variable outcome (13). eHealth solutions provide a promising tool to enable such intervention in a more resource and user friendly manner. So far, limited examples, including the unsuccessful e-moms roc trial, and partially successful SmartMoms intervention, call for more endeavors to identify approach that can consistently deliver positive results $(14,15)$.

Herein, we report an eHealth platform tailored to Chinese pregnant women that was evaluated for its effectiveness and user-experience among pregnant women receiving prenatal care at a private hospital in Beijing. The primary objective of this study is to investigate the effects of Pregnancy Butler eHealth 
intervention on gestational weight gain management. And secondary objectives are to investigate the effects on other clinical outcomes associated with eGWG, e.g., GDM incidence.

\section{Methods}

\section{Study design}

The current study is a randomized trial among participating pregnant women who received prenatal care at Beijing New Century Women's and Children's Hospital (New Century hospital). It was registered as "Pregnancy Butler for gestational weight gain management" (ChiCTR1800014647) under the China Clinical Trial Registry, with approval by the Ethical Committee of Beijing New Century Women's and Children's Hospital (PP/ETC/021711001/01).

\section{Recruitment of subjects}

As the primary endpoint of this study is defined as GWG, the sample size is calculated based on the study conducted by Asbee S $\mathrm{M}$ et al aiming to investigate whether dietary and lifestyle counseling prevents eGWG with a group of subjects without imitation on pre-pregnancy BMI (16). With the differences of $3.1 \mathrm{~kg}$, standard deviation of $6.4 \mathrm{~kg}$, power level of $80 \%$, and an alpha of 0.05 , a minimum of 68 subjects is needed for each group. In addition, considering that historically only $40-50 \%$ of people who conducted early pregnancy checkup at gestational week 5-11 decided to continuously conduct routine prenatal care in New Century hospital later on, around 180 subjects were enrolled for each group.

The overall experimental design is shown in Fig. 1 following the CONSORT standard (17). Rolling recruitment was employed among candidate subjects with confirmed early pregnancy between gestational week 5-11. Eligible candidate subjects were inquired for their willingness to participate, and were recruited if their provided informed consent. Initial inclusion criteria are listed in Table 1. When recruited, each subject was randomly assigned to the intervention or control group. The entire recruitment took 8 months from December in 2017 to August in 2018. 
Table 1

Inclusion and exclusion criteria for subject recruitment.

\section{Inclusion}

Early diagnosis of pregnancy with single fetus

Mandarin speaking and living in Beijing

Willing to participate and decided to commit to

prenatal care at the Beijing New Century

Women's and Children's Hospital

Not enrolled in other studies

Age between 18 and $45 \mathrm{yrs}$

\section{Exclusion from analysis}

Younger than 18 or older than 45 yrs old

With existing condition that may affect weight gain, e.g. diabetes, hypertension, thyroid diseases

Multiparous pregnancy
Ectopic pregnancy

With conditions that can affect fetal development, e.g. growth restriction, distress, umbilical cord or placenta abnormities,

Uses a smartphone with iOS (iPhone 6 and beyond)
Miscarriage or elective abortion

Developed major pregnancy complications that could confound outcome, e.g. preeclampsia.

Preterm labor ( $<37$ week)

Failed to commit to the total prenatal care at the New Century Women's and Children's Hospital

\section{Prenatal care received by the subjects}

Standard prenatal care was delivered to all eligible subjects as included in the antenatal examination package established by the New Century Hospital. This routine care included a total of 11 scheduled visits to the obstetrician, during which data concerning the endpoints highlighted in Table 2 were collected as standard prenatal procedures. One face-to-face nutrition counseling session was also included in the routine prenatal care. There was no extra visit required for either the intervention or control group subjects. The key visits were the first, fifth, and the last, during which baseline information, blood glucose, and pregnancy outcome were measured. Also, questionnaires regarding dietary, physical activity, quality of life, and nutrition awareness status were completed for a total of six times during the entire study period. While the intervention group had eHealth application for body weight tracking, the control group subjects received paper forms to record daily body weights. 
Table 2

Data collected at each of the 11 routine visits as standard prenatal care

\section{Time point (Visit number and gestational week)}

\begin{tabular}{|c|c|c|c|c|c|c|c|c|c|c|c|}
\hline Visits & 1 & 2 & 3 & 4 & 5 & 6 & 7 & 8 & 9 & 10 & 11 \\
\hline Gestation weeks & $\begin{array}{l}5- \\
11\end{array}$ & $\begin{array}{l}11- \\
13\end{array}$ & $\begin{array}{l}13- \\
16\end{array}$ & $\begin{array}{l}17- \\
20\end{array}$ & $\begin{array}{l}21- \\
24\end{array}$ & $\begin{array}{l}25- \\
28\end{array}$ & $\begin{array}{l}29- \\
32\end{array}$ & $\begin{array}{l}33- \\
35\end{array}$ & 36 & 37 & $\begin{array}{l}38- \\
42\end{array}$ \\
\hline Basic info & $\mathrm{x}$ & & & & & & & & & & \\
\hline $\begin{array}{l}\text { Fasting blood } \\
\text { glucose }\end{array}$ & & $x$ & & & $x$ & & & & & & \\
\hline OGTT & & & & & $x$ & & & & & & \\
\hline $\begin{array}{l}\text { Pregnancy } \\
\text { outcome }\end{array}$ & & & & & & & & & & & $x$ \\
\hline \multicolumn{12}{|l|}{ Questionnaires: } \\
\hline$F F Q$ & $\mathrm{x}$ & $x$ & $\mathrm{X}$ & $\mathrm{x}$ & $\mathrm{x}$ & & & & & & $\mathrm{x}$ \\
\hline$P A$ & $\mathrm{x}$ & $x$ & $\mathrm{X}$ & $x$ & $x$ & & & & & & $x$ \\
\hline QoL & $x$ & & & & $\mathrm{x}$ & & & & & & $x$ \\
\hline Nutrition KAP & $x$ & & & & $x$ & & & & & & $x$ \\
\hline User feedback & $x$ & & & & $x$ & & & & & & $x$ \\
\hline \multirow[t]{2}{*}{ Body weight } & \multicolumn{11}{|c|}{ Recommended to record weight twice per week from home } \\
\hline & \multicolumn{11}{|c|}{ Body weight during obstetric visits } \\
\hline
\end{tabular}

\section{Pregnancy Butler eHealth intervention}

Besides standard prenatal care, the intervention group also received the Pregnancy Butler eHealth solution throughout the pregnancy journey, which was developed jointly by Koninklijke Philips (Shanghai, China) and Beijing New Century Women's and Children's Hospital, as a platform with an iPad application for health care providers (HCP) and a user smartphone application for pregnant women.

The HCP application for physicians and dietitians provides a subject dashboard with key parameters, predicted risk, and warning tags (Fig. 2a). The next level of user interface zooms in to individual subjects' prenatal care records, lifestyle data, risk factors, and body weight gain curve versus the recommended IOM range (Fig. 2b). Also, direct coaching and tailored reading content can be pushed via this terminal towards individual subjects. The pregnant women's user interface includes meal plan, food records, reading content, physical activity and body weight dashboard (Fig. 2c). Specifically, for dietary record, the 
users can log in each food item with name and weight, while the platform back end would automatically breakdown to macronutrient, iron, calcium, folate, and caloric information. Dietitian could then use these analytics to adjust meal plans and recipe recommendations based on actual intake, which were pushed towards the users (Fig. 2d).

In addition to this eHealth platform, intervention group subjects were also required to attend 4 additional face-to-face nutrition consultations aiming to manage risk for eGWG.

\section{Subject adherence measurements from the eHealth platform}

The usages of Pregnancy Butler application by individual subjects were recorded throughout the study period, with access log for different features. Frequency of usage, expressed as (total log-in count / number of weeks since registration), and adherence to the application, expressed as (number of weeks during which at least one log-in was recorded/ number of weeks since registration), were used as summary parameters that reflects intervention group subjects' application usage behavior.

\section{Pregnancy outcome measured}

The main clinical outcomes for this study include GWG as primary endpoints, oral glucose tolerance test (OGTT) results, GDM incidence, delivery mode as well as birth weight as secondary endpoints. These data were largely extracted directly from the hospital's information system as part of health record. In addition, participating subjects were recommended to record body weight regularly using their own scales at home, with records submitted either through Pregnancy Butler application, or hardcopy sheets for those in the control group. These records were considered together with in-hospital body weight measurements as dynamic trend of weight gain. Some of the outcomes were analyzed around the end of the second trimester ( week 26 ), especially GDM related. The rest were only considered at the end of pregnancy.

\section{Questionnaires}

As indicated in Table 1, the subjects were asked to answer four questionnaires during 5 of their routine prenatal care visits during the first and second trimesters, with a final revisit to the questions towards the end of pregnancy. While dietary and physical activity survey were conducted for 6 times in total for each subject, quality of life (QoL) and nutrition awareness were sampled for three times only.

In this study, Food frequency questionnaire (FFQ) was applied for an approximation of usual intake in different pregnancy trimesters. The FFQ used was modified based on the questionnaire developed for the 2011 China Nutrition and Health Survey (CHNS) (18). In this particular case, alcohol and tea drinks were removed from the food list.

Physical activity level was estimated with a questionnaire on type, frequency, and duration of physical exercises or activities adopted and modified from Zhang et al. (19). 
Quality of life was assessed by requesting the subjects to provide a subjective score on a scale of 0 to 100 regarding their quality of life, similar to previously reported (20).

To assess nutrition knowledge and behavior awareness, the nutrition knowledge, attitude and practice questionnaire developed by Hu et al. for Chinese pregnant women was used in this study after modification(21).

Feedback on user experience was also collected among intervention group subjects for three times across the study, focusing on subjective rating of the attractiveness of different features of the eHealth solution on a scale of $1-5$. Although it was highly recommended to all participating subjects, feedback questionnaire was not mandatory so the length of stay for prenatal visit could be better managed.

\section{Statistical analysis}

All data was archived after de-identification and matched with unique anonymous subject I.D. before analysis. For clinical data, numerical parameters were analyzed using student's t-test for between-group comparisons, after confirmation of normality and equal variance. Categorical parameter comparisons were conducted using Chi-square tests. When it comes to repeatedly measured endpoints such as GWG and questionnaire result evaluation, mixed generalized linear model (GLM) was employed. All statistical analysis was done using R (version 3.5.1 Feather Spray, https://www.R-project.org), with plotting enabled by either Microsoft Excel 2016 (Microsoft, WA, US) or the ggplot2 package in R.

\section{Results}

\section{Study subject profile}

As described in Fig. 1, there were a total of 227 and 207 subjects were eligible for week 26 and final endpoint analysis, respectively. The intervention group started with 16 more subjects to the point of official sign up for prenatal care (second visit around week 12), mainly due to more subjects in the control group decided to conduct routine prenatal care in other hospitals.

Table 3 illustrates the demographic profile of subjects subjected to data analysis. The average age was 32.1 years old for all of the participants, with a BMI of $21.1 \mathrm{~kg} / \mathrm{m}^{2}$. Approximately $61 \%$ of them were pregnant for the first time, with almost all the rest having the second child. Household income and education background revealed a group with high social-economic status, which is expected provided that they were customers of private hospital. There was no apparent difference between the control and intervention group in any of the parameters, indicating a balanced baseline resulted from the recruitment. 
Table 3

Baseline demographic characteristics of eligible subjects in week 26

\begin{tabular}{|c|c|c|c|c|c|c|c|c|}
\hline & & \multicolumn{3}{|c|}{ Control } & \multicolumn{3}{|c|}{ Intervention } & \multirow[t]{2}{*}{$\begin{array}{l}\text { P-value for } \\
\text { difference }\end{array}$} \\
\hline Total number $(\mathrm{N}=)$ & & 106 & & & 121 & & & \\
\hline Age & (Year) & 32.0 & \pm & 3.6 & 32.2 & \pm & 3.7 & 0.397 \\
\hline Pre-pregnancy BMI & $\left(\mathrm{kg} / \mathrm{m}^{2}\right)$ & 21.1 & \pm & 2.9 & 21.2 & \pm & 3.0 & 0.295 \\
\hline BMI classification & Underweight & 15 & & $14 \%$ & 17 & & $14 \%$ & 0.443 \\
\hline \multirow[t]{3}{*}{ (Chinese criteria)* } & Normal & 78 & & $74 \%$ & 85 & & $70 \%$ & \\
\hline & Overweight & 7 & & $7 \%$ & 14 & & $12 \%$ & \\
\hline & Obese & 6 & & $6 \%$ & 5 & & $4 \%$ & \\
\hline $\begin{array}{l}\text { Pre-pregnancy } \\
\text { weight }\end{array}$ & ( $\mathrm{kg})$ & 56.7 & \pm & 8.4 & 57.6 & \pm & 9.0 & 0.560 \\
\hline \multirow[t]{4}{*}{ Parity } & $1 s t$ & 66 & & $62 \%$ & 71 & & $60 \%$ & 0.987 \\
\hline & $2 n d$ & 36 & & $35 \%$ & 45 & & $37 \%$ & \\
\hline & $3 r d$ & 2 & & $2 \%$ & 2 & & $2 \%$ & \\
\hline & 4th & 1 & & $1 \%$ & 1 & & $1 \%$ & \\
\hline \multirow[t]{3}{*}{ Education } & $\begin{array}{l}\text { Did not } \\
\text { disclose }\end{array}$ & 5 & & $5 \%$ & 5 & & $4 \%$ & 0.751 \\
\hline & $\begin{array}{l}\text { Without } \\
\text { College }\end{array}$ & 1 & & $1 \%$ & 1 & & $1 \%$ & \\
\hline & $\begin{array}{l}\text { College or } \\
\text { above }\end{array}$ & 100 & & $94 \%$ & 115 & & $95 \%$ & \\
\hline Household income & $\begin{array}{l}\text { Did not } \\
\text { disclose }\end{array}$ & 2 & & $2 \%$ & 2 & & $2 \%$ & 0.298 \\
\hline \multirow[t]{4}{*}{ (CNY/mo..) } & $<10,000$ & 5 & & $5 \%$ & 6 & & $5 \%$ & \\
\hline & $10,000-20,000$ & 20 & & $19 \%$ & 33 & & $28 \%$ & \\
\hline & $20,000-30,000$ & 32 & & $30 \%$ & 27 & & $23 \%$ & \\
\hline & $>30,000$ & 46 & & $44 \%$ & 51 & & $43 \%$ & \\
\hline $\begin{array}{l}*: 18.5 \leq \mathrm{BMI}<24.0: \\
\geq 28.0\end{array}$ & 1I < 18.5; Normal: & $8.5 \leq E$ & & $4.0 ; 0$ & rweigh & & $0 \leq B N$ & < 28.0; Obese: BMI \\
\hline
\end{tabular}

\section{Adherence to intervention}


Subjects were able to provide body weight records, either through the eHealth application if they were in the intervention group, or on hard copy weight record sheets for those in the control group. Intervention group subjects were monitored for their access and usage of the application, with the results shown in Fig. 3. As defined in the methods, two parameters were used to reflect engagement to the eHealth application. The usage intensity, shown as average usage per week, showed high variability among users, with a median of 2.0 times/per week (Fig. 3a). There was an apparent increasing trend of usage in the early stage during the 1 st trimester, reaching an average of 5 times per week. Afterwards, intensity gradually dropped to a steady level until the end of pregnancy. While some subjects showed good sustained engagement to the application, a large number of them maintained relatively low usage intensity. The other adherence parameter was cumulative active rate, which is defined as the proportion of weeks during pregnancy with at least one usage of the Pregnancy Butler application. There was a descent in active rate through the early second trimester, after which a plateau was reached around a median of 0.5 that maintained towards delivery (Fig. $3 \mathrm{c}$ ). This means that on average, a subject used the application every other week. More even distribution of active rate than usage level among subjects is shown in Fig. 3d.

The reception of the Pregnancy Butler was also positive among the users, with an average rating of 3.8 out of 5 at around week 12 , which slightly dropped to 3.4 by around week 25 . The average rating of ease of use was 3.6 out of 5 .

\section{Clinical pregnancy outcome}

Table 4 summarizes the clinical pregnancy outcome of the study cohort. There was $36 \%$ and $34 \%$ of subjects gained excessive weight, for the control and intervention groups respectively. While $25 \%$ of control group subjects were diagnosed with GDM, the incidence was $21 \%$ for those in the eHealth intervention group. However, the difference was not statistically significant ( $p$-value $=0.70)$. There was also no difference between the two groups in delivery mode or absolute birth weight. However, higher number of macrosomia cases was noted in the intervention group. 
Table 4

Clinical pregnancy outcome for all eligible subjects in week 26 and final endpoint

\begin{tabular}{|c|c|c|c|c|c|c|c|c|}
\hline & & \multicolumn{3}{|c|}{ Control } & \multicolumn{3}{|c|}{ Intervention } & \multirow{2}{*}{$\begin{array}{l}\text { P-value } \\
\text { for } \\
\text { difference }\end{array}$} \\
\hline \multicolumn{2}{|l|}{$\begin{array}{l}\text { Subject number (week 26/final } \\
\text { endpoint) }\end{array}$} & \multicolumn{3}{|c|}{$106 / 94$} & \multicolumn{3}{|c|}{$121 / 113$} & \\
\hline Weight gain at OGTT & $\mathrm{kg}$ & 6.7 & \pm & 2.9 & 6.7 & \pm & 2.6 & $0.849 *$ \\
\hline Delivery weight gain & & 14.4 & \pm & 4.3 & 14.3 & \pm & 4.2 & $0.901 *$ \\
\hline \multirow[t]{3}{*}{ Delivery GWG class } & Low & 18 & & $19 \%$ & 20 & & $18 \%$ & \multirow[t]{3}{*}{$0.879^{\wedge}$} \\
\hline & Normal & 42 & & $45 \%$ & 54 & & $48 \%$ & \\
\hline & Excessive & 34 & & $36 \%$ & 38 & & $34 \%$ & \\
\hline \multirow[t]{2}{*}{ GDM diagnosis } & Positive & 26 & & $25 \%$ & 26 & & $21 \%$ & \multirow[t]{2}{*}{$0.700^{\wedge}$} \\
\hline & Negative & 80 & & $75 \%$ & 95 & & $79 \%$ & \\
\hline \multirow[t]{2}{*}{ Delivery mode } & Natural & 23 & & $24 \%$ & 33 & & $29 \%$ & \multirow[t]{2}{*}{$0.544^{\wedge}$} \\
\hline & C-section & 71 & & $76 \%$ & 80 & & $71 \%$ & \\
\hline \multirow[t]{2}{*}{ Birth weight } & $g$ & 3348 & \pm & 445 & 3450 & \pm & 399 & $0.087^{\star}$ \\
\hline & $\geq 4 \mathrm{~kg}$ & 3 & & $3 \%$ & 13 & & $11 \%$ & $0.049^{\wedge}$ \\
\hline \multicolumn{9}{|c|}{ Shown as mean \pm S.D. or count with percentage of total; } \\
\hline \multicolumn{9}{|c|}{ *: Student's t-test; ${ }^{\wedge}$ : Chi-square test. } \\
\hline
\end{tabular}

Since pre-pregnancy body weight is a well-established risk for metabolic abnormities during pregnancy, outcome analysis was also done among those being overweight or obese (BMI $\geq 24 \mathrm{~kg} / \mathrm{m}^{2}$ ), as shown in Table 5. While both groups had comparable yet small proportion of subjects being overweight or obese before pregnancy, there was lower incidence of eGWG ( $p$-value $=0.0496)$ among those who received the eHealth intervention. While the chi-square test did not show low p-value due to small sample size, there was evidently lower GDM incidence in the intervention group. The average absolute weight gain was not significantly different between the two groups, despite $1.4 \mathrm{~kg}$ of absolute discrepancy in mean at the time of delivery. Interestingly, there was a trend showing higher C-section rate among overweight and obese subjects in the control group. 
Table 5

Clinical pregnancy outcome for subjects with pre-pregnancy BMI $\geq 24 \mathrm{~kg} / \mathrm{m}^{2}$.

\begin{tabular}{|c|c|c|c|c|c|c|c|c|}
\hline & & Contro & & & Interve & ntion & & P-value for difference \\
\hline Subject number & & 13 & & & 19 & & & $0.304^{\wedge}$ \\
\hline Weight gain at OGTT & \multirow[t]{2}{*}{$k g$} & 5.4 & \pm & 5.0 & 4.5 & \pm & 3.0 & $0.579 *$ \\
\hline Delivery weight gain & & 13.0 & \pm & 4.6 & 11.6 & \pm & 5.7 & $0.542^{*}$ \\
\hline \multirow[t]{3}{*}{ Delivery GWG class } & Low & 3 & & $23 \%$ & 2 & & $11 \%$ & \multirow[t]{3}{*}{$0.0496^{\wedge}$} \\
\hline & Normal & 0 & & $0 \%$ & 7 & & $37 \%$ & \\
\hline & Excessive & 10 & & $77 \%$ & 10 & & $53 \%$ & \\
\hline \multirow[t]{2}{*}{ GDM diagnosis } & Positive & 7 & & $54 \%$ & 7 & & $36 \%$ & \multirow[t]{2}{*}{$0.556^{\wedge}$} \\
\hline & Negative & 6 & & $46 \%$ & 12 & & $64 \%$ & \\
\hline \multirow[t]{2}{*}{ Delivery mode } & Natural & 8 & & $62 \%$ & 17 & & $89 \%$ & \multirow[t]{2}{*}{$0.149^{\wedge}$} \\
\hline & C-section & 5 & & $38 \%$ & 2 & & $11 \%$ & \\
\hline \multirow[t]{2}{*}{ Birth weight } & $g$ & 3338 & \pm & 448 & 3478 & \pm & 503 & $0.426^{*}$ \\
\hline & $>4 \mathrm{~kg}$ & 1 & & $8 \%$ & 3 & & $16 \%$ & $0.892^{\wedge}$ \\
\hline
\end{tabular}

Shown as mean \pm S.D. or count with percentage of total;

Student's t-test; ${ }^{\wedge}$ : Chi-square test

\section{Weight gain dynamic through the pregnancy period}

While the single time-point of GWG did not separate between the control and intervention groups, a timecourse view also showed similar weight gain profile between subjects in the control and intervention groups ( $p$-value for incremental area under curve (iAUC) $=0.180$, Fig. $4 a$ ). On the other hand, there was an apparent and consistent trend of lower weight gain in overweight/obese subjects who received the Pregnancy Butler intervention, despite a borderline p-value of 0.079 for iAUC comparison (Fig. 4b).

\section{Impact of eHealth engagement level on clinical outcome}

Given that the clinical outcome of lifestyle intervention often depends on the level of engagement, the weight gain profile of active users and inactive users, divided by the median usage level described earlier, were compared with each other, as shown in Fig. 5. The active users who had higher frequency of access to the Pregnancy Butler application consistently showed lower absolute weight gain (iAUC p-value = 0.047 ) and more favorable GWG classification ( $p$-value $=0.022$ ). There was no baseline different between active and inactive users regarding background demographic or biometric parameters (data not shown).

\section{Association between gestational weight gain and GDM}


With the high incidence of eGWG and GDM among the study subjects, the mutual interaction between the two conditions were characterized as in Table 6, subjects with GDM were more likely to end up with lower than desired or excessive weight gain.

Table 6

Association between GDM diagnosis and GWG outcome among study subjects.

\begin{tabular}{|c|c|c|c|c|c|c|}
\hline & & \multicolumn{2}{|c|}{ Without GDM } & \multicolumn{2}{|c|}{ With GDM } & \multirow[t]{2}{*}{$\mathrm{p}$-value for difference } \\
\hline Total number & Delivered & & 157 & & & \\
\hline \multirow[t]{3}{*}{ GWG classification } & Low & 26 & $17 \%$ & 15 & $30 \%$ & \multirow[t]{3}{*}{0.017} \\
\hline & Normal & 78 & $50 \%$ & 16 & $32 \%$ & \\
\hline & Excessive & 53 & $33 \%$ & 19 & $38 \%$ & \\
\hline
\end{tabular}

\section{Predictors for eGWG and GDM risks}

Although eHealth intervention is the focus of this current study, early detection of metabolic risk during pregnancy was also a feature integrated as part of the Pregnancy Butler solution.

Pre-pregnancy BMI showed good predictive value for eGWG, as indicated by Fig. 6a. Subjects who were overweight or obese before pregnancy had drastically higher risk to gain excessive weight, with an odds ratio (OR) of 3.6 (95\% Cl: $1.6 \sim 8.3$ ) over normal weight subjects. Also, when gaining more than recommended weight by 12 weeks of pregnancy, the OR for being eGWG at the end of pregnancy was 8.9 (95\% Cl: $2.9 \sim 27.5)$.

For GDM, at-risk subjects were defined as either older than 35 or being overweight / obese. On average, the incidence of GDM was $22.9 \%$ among eligible subjects. As shown in Fig. $6 \mathrm{~b}$, the at-risk subjects had overwhelmingly high risk for developing GDM, with an OR of 15.1 (95\% Cl: $6.9 \sim 32.9$ ).

\section{Lifestyle behavior pattern}

Based on questionnaire survey results, there appeared to be no significant differentiation between the intervention group and control group, regarding dietary quality score, physical activity score, or KAP score on healthy lifestyle (data not shown).

\section{Discussions}

The current study explored an eHealth intervention against pregnancy-associated metabolic risks, specifically eGWG. The reception of the Pregnancy Butler was positive among the users, especially at the beginning. However, the usage level slumped after the initial excitement to a rather moderate level, which was more evident among inactive users. While the engagement level of the current study was better than the e-moms roc trial reported by Olson et al., it remained shy of anticipated as indicated by the nonsignificant improvement in diet or physical activity (14). 
The key clinical outcome parameters including GWG, GDM incidence, delivery mode and birth weight, did not show superiority with the eHealth intervention among the whole participants, which could at least be partially attributed to the lower than desired engagement level. This is supported by the fact that active users, with more intensive usage of the application, had lower risk in gaining excessive weight. Besides, there was higher rate of macrosomia in the intervention group. Further analysis found that the incidence of macrosomia in the control group was not significantly different from that of active users in the intervention group ( $p$-value $=0.487$ ), while both were lower than the incidence in the inactive users who couldn't meet the median engagement level ( $p$-value $=0.002,0.039$, respectively). In order for internet technology based eHealth platform to receive more than desired intervention effects, it is worth considering to modify the application to an easier to use manner for better user engagement ad adherence. Function simplification and workflow modification, e.g. reducing the levels of menu choices needed to navigate through for weight and diet recording, to reduce the efforts and burdens of pregnant women could be potential improvement directions.

The other potential factor that might have undermined the intervention effect is the high level of nonmodifiable risks for eGWG or GDM carried by the subjects. Since this study was conducted at a private hospital, the subjects held significantly higher than average age, education level, and social-economic status than general Chinese pregnant women $(22,23)$. The advanced age could have predisposed the subject group to high risk for eGWG and GDM, as indicated by the overall incidence at the end of the study $(24,25)$. The weight gain profile of the studied subjects showed some striking difference from the reported norm in China, with more rapid growth in the second and third trimester (26).

On the other hand, the post-hoc analysis of the intervention effect among overweight or obese subjects in the current study showed an apparent trend of lower weight gain, despite the small sub-sample size. In addition, there were implications of reduced GDM incidence and C-section rate for overweight or obese subjects in the intervention group. However, as a modifiable risk, overweight and obesity accounting for only $12 \%$ of the total subjects, which made it difficult to improve the overall eGWG and GDM outcome, as most successful intervention trials were done in obese or even morbidly obese individuals only (27-29). A Cochrane report also indicated that subject profile could affect intervention outcome, together with intervention engagement, while there is suggestive evidence that supports the efficacy of such interventions (13). It should be more effective if future interventions can be applied only to those with preexisting metabolic risk factors, rather than overall healthy pregnant women $(23,30)$.

The main limitation of this study is that for control group, besides the standard prenatal care, subjects received paper forms to record daily body weights for body weight tracking, which might change their regular home weighing routine to a great extent, as well as rise their awareness of weight control and lifestyle change, which may further affect the clinical outcomes.

From insight perspective, one phenomena observed could be of clinical value, that is the importance of early intervention, even before pregnancy, to minimize eGWG risk. Our data indicated several folds higher risk of eGWG for those with higher than $24 \mathrm{~kg} / \mathrm{m}^{2} \mathrm{BMI}$ or gained extra weight in the first trimester, as well 
as $>50 \%$ risk of diagnosed with GDM for those with advanced age ( $>35)$ or overweight only. This is in line with recent discussions on early engagement and intervention against pregnancy metabolic risks (31, 32).

\section{Conclusion}

The current study showed that Pregnancy Butler, our exploratory eHealth intervention against pregnancy metabolic dysregulations, was well accepted and moderately utilized by the pregnant subjects in a private obstetric hospital in China. However, the overall usage and unfavorable risk factors compromised general clinical benefit, without improving overall outcome. Nevertheless, its effectiveness was still present among overweight and obese subjects, whose eGWG incidence was lowered by the intervention. Future studies and applications of such eHealth platforms could focus more on high metabolic risk population, with more emphasis on behavioral change before or early in pregnancy through improved easiness to use, user interaction, and engagement tactics.

\section{Abbreviations}

$\mathrm{BMI}$

body mass index

CHNS

China Nutrition and Health Survey

eGWG

excessive gestational weight gain

FFQ

Food frequency questionnaire

GDM

gestational diabetes mellitus

GLM

generalized linear model

GWG

gestational weight gain

$\mathrm{HCP}$

health care providers

IOM

Institute of Medicine

OGTT

oral glucose tolerance test

QoL

Quality of life 


\section{Declarations}

\section{Ethics approval and consent to participate}

This study was approved by the Ethical Committee of Beijing New Century Women's and Children's Hospital (PP/ETC/021711001/01) in Nov 29, 2017. Informed consents to participate in the study were obtained from participants.

\section{Consent for publication}

Not applicable

\section{Availability of data and materials}

The datasets used and/or analysed during the current study are available from the corresponding author on reasonable request.

\section{Competing interests}

Financial support was jointly by Koninklijke Philips and the New Century Healthcare Group. Philips owns connected care solution business. The following authors are employees of Philips: N.H., Q.H., Y.J., H.M., Y.Y., and X.Z. New Century Healthcare Group owns private hospitals with prenatal care service. The following authors are employees of New Century Health care Group: X.W., D.Z., G.L., L.F., G.S. and J.L.

\section{Funding}

This study was funded jointly by Koninklijke Philips and the New Century Healthcare Group.

\section{Authors' contributions}

X.W., Q.H., D.Z. and G.L. designed the study. X.W., D.Z., G.L., L.F., G.S. and J.L. carried out the study. N.H., Q.H., Y.J., H.M., Y.Y., and X.Z. analyzed the de-identified data. X.W., and N.H. wrote the manuscript. G.L. had primary responsibility for final content. All authors read and approved the final manuscript.

\section{Acknowledgements}

We express our gratitude to the following contributors for their assistance in the successful implementation of this study: Zhenzhen Cai, Yu Qi, Lin Li, Yuqiang Wu, Xiaoming Zhou, Szuying Ching, Wenxi Wang, and Juming Tang.

\section{References}

1. Huang A, Ji Z, Zhao W, Hu H, Yang Q, Chen D. Rate of gestational weight gain and preterm birth in relation to prepregnancy body mass indices and trimester: A follow-up study in China. Reprod Health. 2016;13(1):1-7. 
2. Koh H, Ee TX, Malhotra R, Allen JC, Tan TC, Østbye T. Predictors and adverse outcomes of inadequate or excessive gestational weight gain in an Asian population. J Obstet Gynaecol Res. 2013;39(5):905-13.

3. Tam CHT, Ma RCW, Yuen LY, Ozaki R, Li AM, Hou Y, et al. The impact of maternal gestational weight gain on cardiometabolic risk factors in children. Diabetologia. 2018;2539-48.

4. Fleming TP, Watkins AJ, Velazquez MA, Mathers JC, Prentice AM, Stephenson J, et al. Origins of lifetime health around the time of conception: causes and consequences. Lancet. 2018;391(10132):1842-52.

5. Miao M, Dai M, Zhang Y, Sun F, Guo X, Sun G. Influence of maternal overweight, obesity and gestational weight gain on the perinatal outcomes in women with gestational diabetes mellitus. Sci Rep. 2017;7(1):1-8.

6. Samura T, Steer J, Daniela Michelis L, Carroll L, Holland E, Perkins R. Factors associated with excessive gestational weight gain: Review of Current Literature. Glob Adv Heal Med. 2016;5(1):8793.

7. Kinnunen TI, Waage CW, Sommer C, Sletner L, Raitanen J, Jenum AK. Ethnic Differences in Gestational Weight Gain: A Population-Based Cohort Study in Norway. Matern Child Health J. 2016;20(7):1485-96.

8. Shin D, Lee KW, Song WO. Dietary Patterns During Pregnancy are Associated with Gestational Weight Gain. Matern Child Health J. 2016;20(12):2527-38.

9. Jiang H, Qian X, Li M, Lynn H, Fan Y, Jiang H, et al. Can physical activity reduce excessive gestational weight gain? Findings from a Chinese urban pregnant women cohort study. Int J Behav Nutr Phys Act. 2012;9:1-7.

10. Asbee SM, Jenkins TR, Butler JR, White J, Elliot M, Rutledge A. Preventing excessive weight gain during pregnancy. Obstet Gynecol. 2009;113(2):305-12.

11. Phelan S, Wing RR, Brannen A, McHugh A, Hagobian TA, Schaffner A, et al. Randomized controlled clinical trial of behavioral lifestyle intervention with partial meal replacement to reduce excessive gestational weight gain. Am J Clin Nutr. 2018;107(2):183-94.

12. Luo XD, Dong X, Zhou J. Effects of nutritional management intervention on gestational weight gain and perinatal outcome. Saudi Med J. 2014;35(10):1267-70.

13. B MC,E, H JT,S. P. M. Diet and exercise interventions for preventing gestational diabetes mellitus-a cochrane review. J Paediatr Child Health. 2014;50(SUPPL. 1):44.

14. Olson CM, Groth SW, Graham ML, Reschke JE, Strawderman MS, Fernandez ID. The effectiveness of an online intervention in preventing excessive gestational weight gain: The e-moms roc randomized controlled trial. BMC Pregnancy Childbirth. 2018;18(1):1-11.

15. Redman LM, Gilmore LA, Breaux J, Thomas DM, Elkind-Hirsch K, Stewart T, et al. Effectiveness of SmartMoms, a Novel eHealth Intervention for Management of Gestational Weight Gain: Randomized Controlled Pilot Trial. JMIR mHealth uHealth. 2017;5(9):e133. 
16. Asbee SM, Jenkins TR, Butler JR, White J, Elliot M, Rutledge A. Preventing excessive weight gain during pregnancy through dietary and lifestyle counseling: a randomized controlled trial. Obstet Gynecol. 2009;113(2):305-12.

17. Moher D, Schulz KF, Altman DG, CONSORT GROUP (Consolidated Standards of Reporting Trials). The CONSORT statement: revised recommendations for improving the quality of reports of parallel-group randomized trials. Ann Intern Med. 2001 Apr;134(8):657-62.

18. Zang J, Luo B, Chang S, Jin S, Shan C, Ma L, et al. Validity and reliability of a food frequency questionnaire for assessing dietary intake among Shanghai residents. Nutr J. 2019 Dec;18(1):30.

19. Zhang Y, Dong S, Zuo J, Hu X, Zhang H, Zhao Y. Physical Activity Level of Urban Pregnant Women in Tianjin, China: A Cross-Sectional Study. Barengo NC, editor. PLoS One. 2014 Oct;9(10):e109624.

20. Ming W-K, Wu H, Wu Y, Chen H, Meng T, Shen Y, et al. Health-related quality of life in pregnancy with uterine fibroid: a cross-sectional study in China. Health Qual Life Outcomes. 2019 Dec;17(1):89.

21. Hu Q. Development and application of Pregnant Women Nutrition KnowlegdeãaAttitude and Practice Questionnaire. Anhui Medical University; 2011.

22. Liu Y, Dai W, Dai X, Li Z. Prepregnancy body mass index and gestational weight gain with the outcome of pregnancy: A 13-year study of 292,568 cases in China. Arch Gynecol Obstet. 2012;286(4):905-11.

23. Chen Z, Du J, Shao L, Zheng L, Wu M, Ai M, et al. Prepregnancy body mass index, gestational weight gain, and pregnancy outcomes in China. Int J Gynecol Obstet. 2010;109(1):41-4.

24. Huang X, Tan H, Cai M, Shi T, Mi C, Lei J. Gestational weight gain in Chinese women -- Results from a retrospective cohort in Changsha, China. BMC Pregnancy Childbirth. 2018;18(1):1-9.

25. Shian Y, Diseases C, Control D, Shian Y. The ten-year retrospect of nutrition and health status of pregnant women in China. Chin J Prev Med. 2018;52(1):94-100.

26. Zhang Y, Wang L, Yin C, Mu K, Niu D, Yan W. Development and evaluation of percentile distribution of body weight by gestational week as a tool for gestational weight management: a retrospective study based on hospital routine data. BMJ Open. 2018;8(6):e019645.

27. Sun $Y$, Zhao $H$. The effectiveness of lifestyle intervention in early pregnancy to prevent gestational diabetes mellitus in Chinese overweight and obese women: A quasi-experimental study. Appl Nurs Res. 2016;30:125-30.

28. Liu YQ, Liu Y, Hua Y, Chen XL. Effect of diet and exercise intervention in Chinese pregnant women on gestational weight gain and perinatal outcomes: A quasi-experimental study. Appl Nurs Res. 2017;36:50-6.

29. Herring SJ, Cruice JF, Bennett GG, Rose MZ, Davey A, Foster GD. Preventing excessive gestational weight gain among African American women: A randomized clinical trial. Obesity. 2016 Jan;24(1):30-6.

30. Oostdam N, van Poppel MNM, Wouters MGAJ, van Mechelen W. Interventions for Preventing Gestational Diabetes Mellitus: A Systematic Review and Meta-Analysis. J Women's Heal. 2011 Oct;20(10):1551-63. 
31. Poon LC, David Mclntyre H, Hyett JA, Fonseca EB da, Hod M. The first-trimester of pregnancy - a window of opportunity for prediction and prevention of pregnancy complications and future life. Diabetes Res Clin Pract. 2018 May;0(0).

32. Barker M, Dombrowski SU, Colbourn T, Fall CHD, Kriznik NM, Lawrence WT, et al. Intervention strategies to improve nutrition and health behaviours before conception. Lancet. 2018;391(10132):1853-64.

\section{Figures}




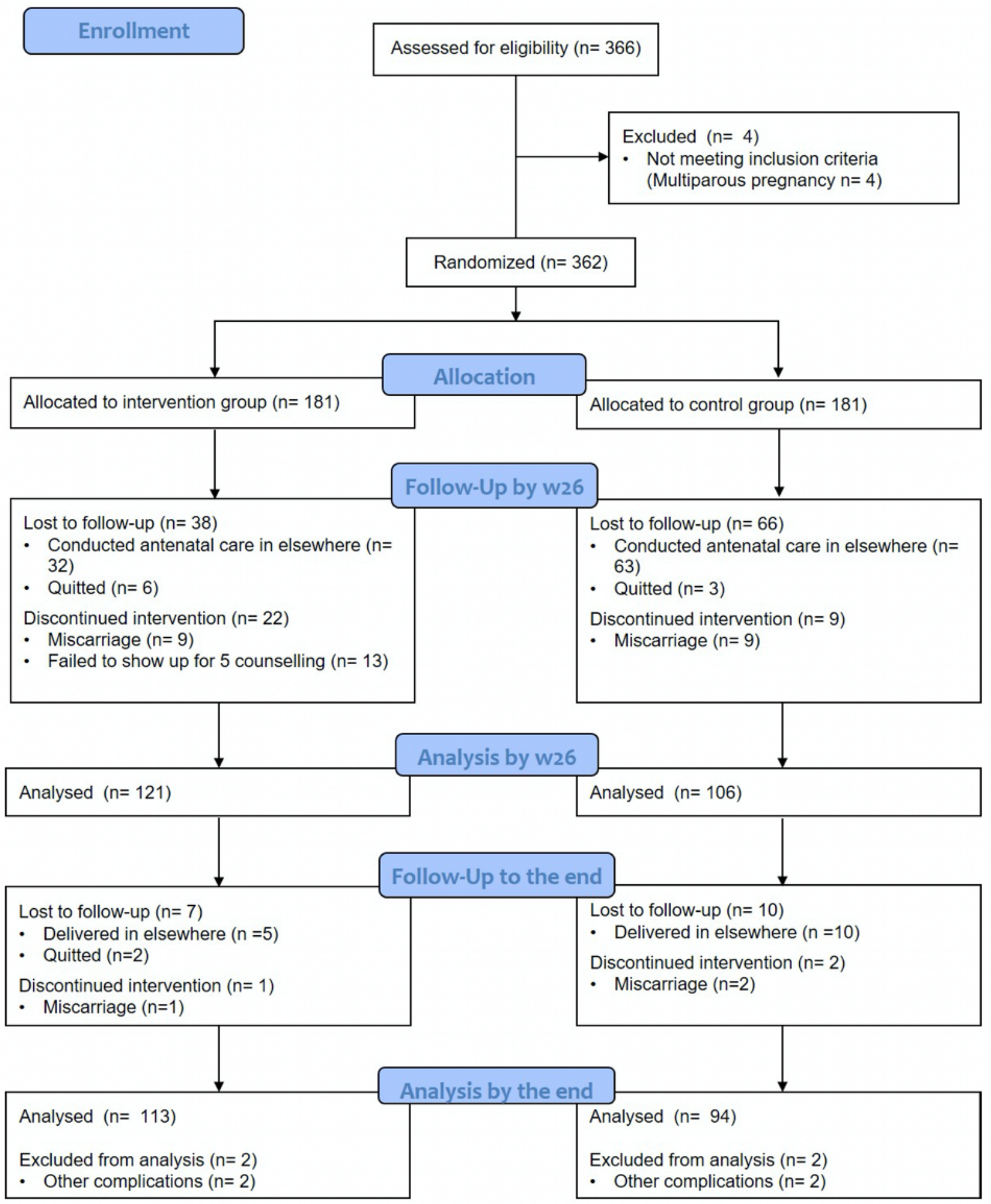

\section{Figure 1}

Flow diagram of the study process (CONSORT) 
a.

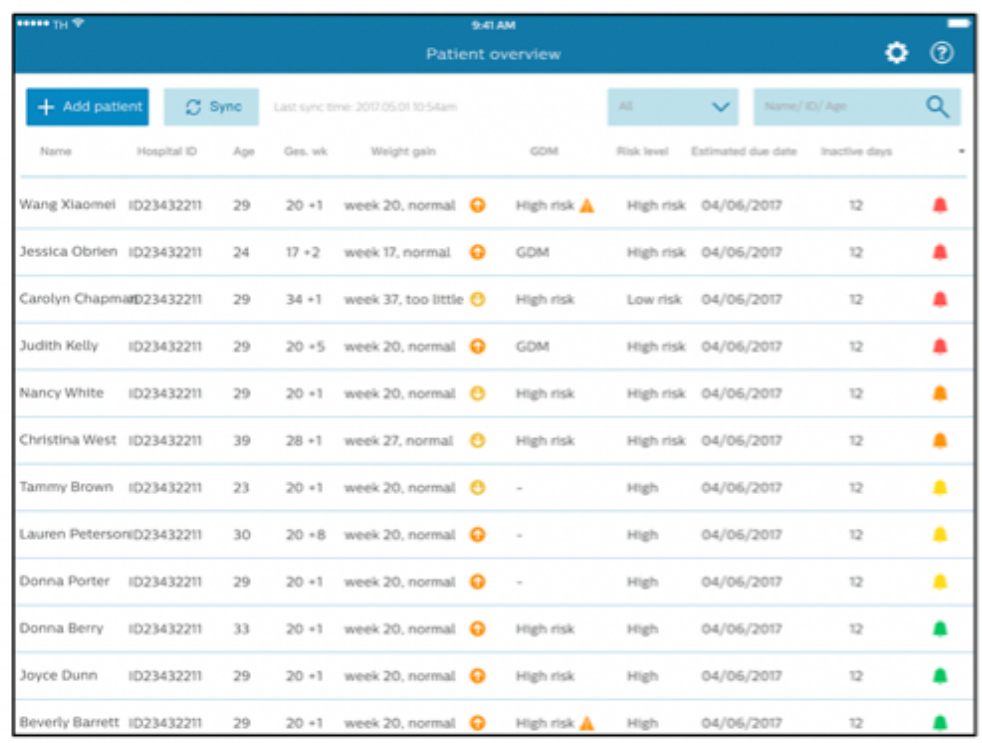

C.

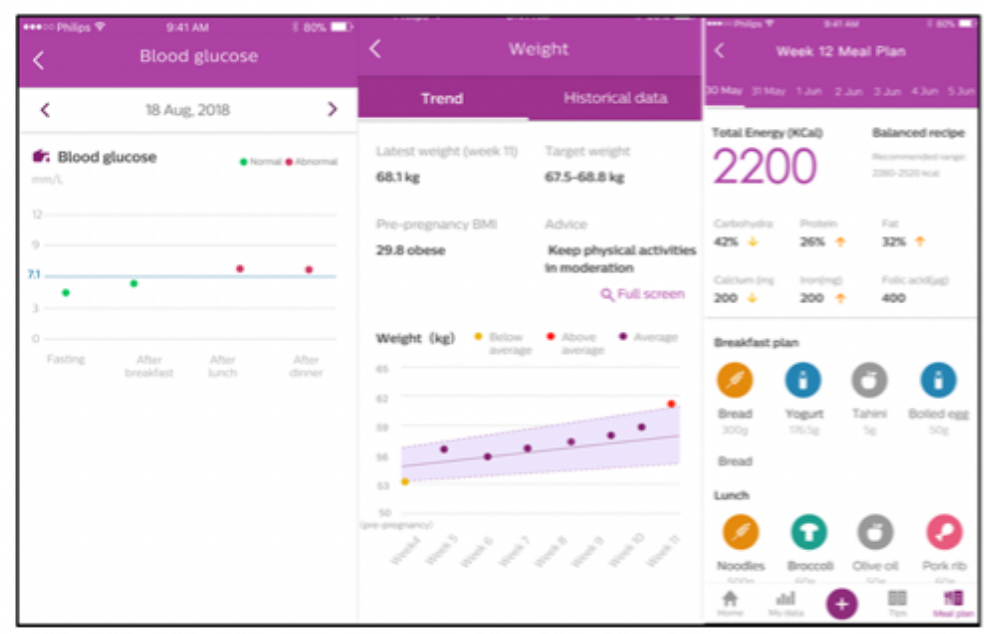

b.

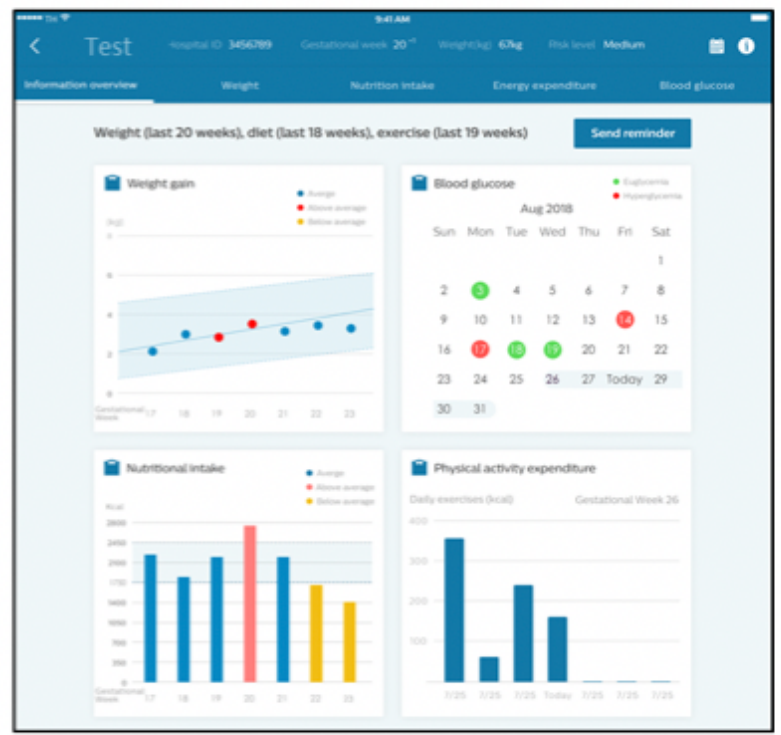

d.

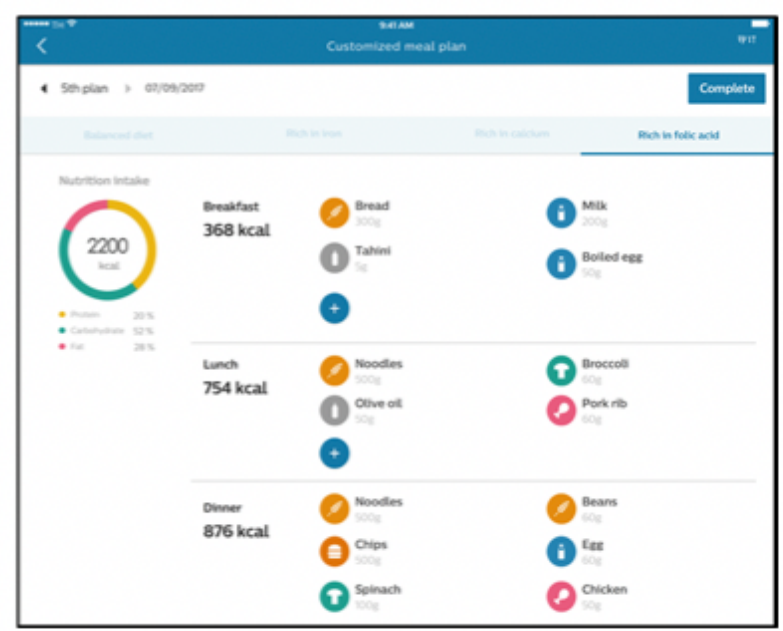

Figure 2

The user interfaces of Pregnancy Butler eHealth platform with simulated user information. (a: HCP dashboard; b: HCP view on specific subject; c: subject/pregnant women dashboard; d: HCP view on dietary plan and record) 
a.

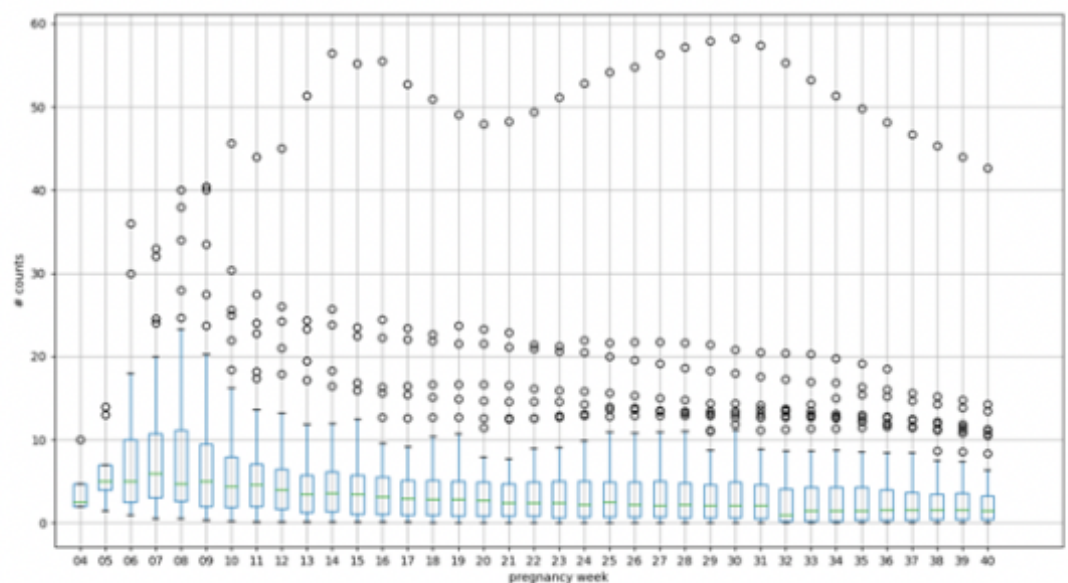

c.

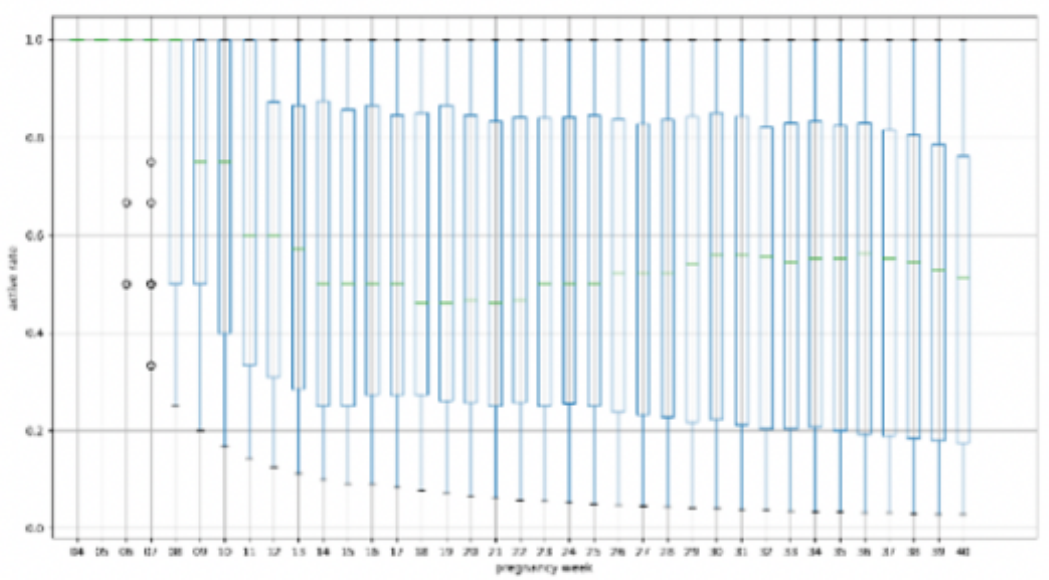

b.

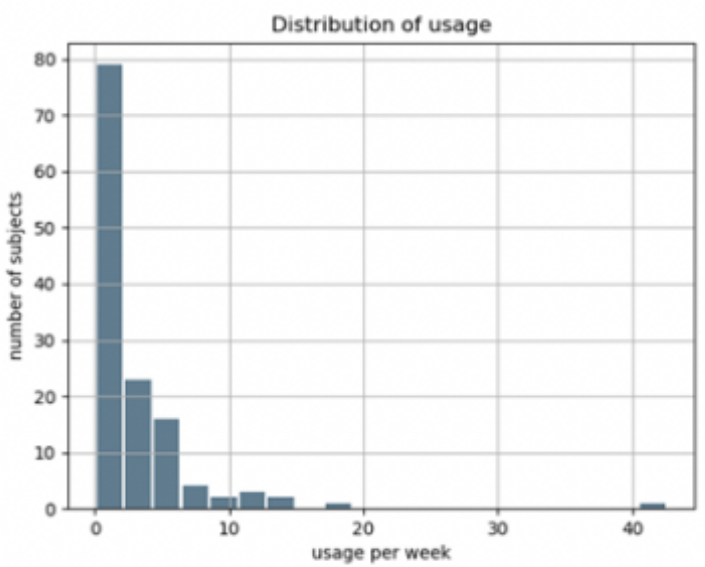

d.

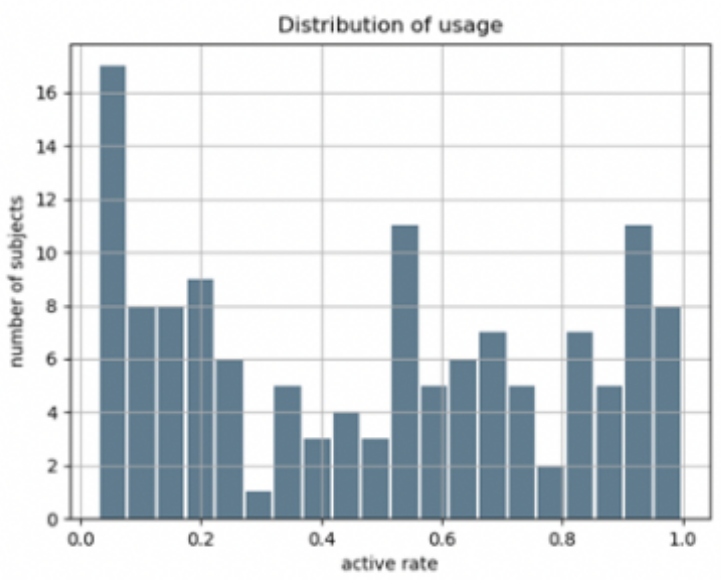

Figure 3

Usage of the eHealth application by subjects in the intervention group. (a: average usage per week through different pregnancy weeks as boxplot; $b$ : distribution of average usage per week by the end of delivery; c: cumulative active rate through different pregnancy weeks as box plots; d: distribution of cumulative active rate by the time of delivery) 
a.

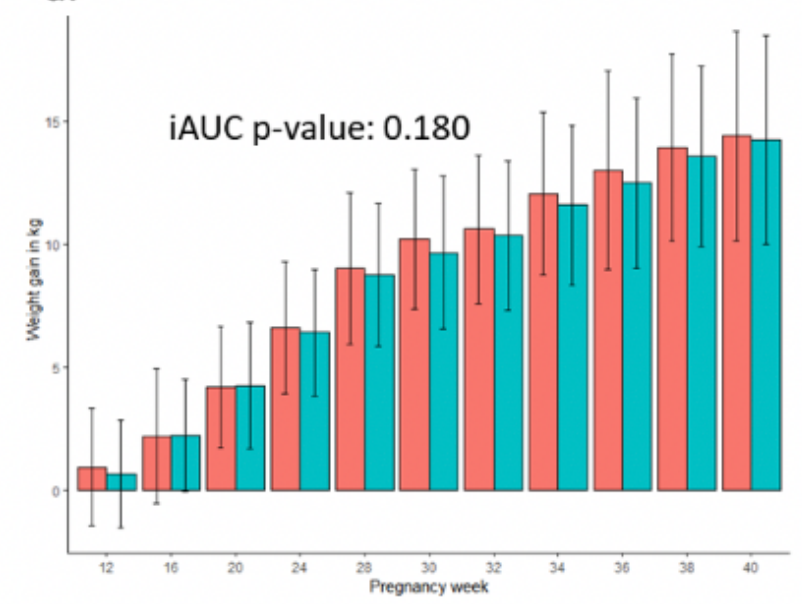

b.

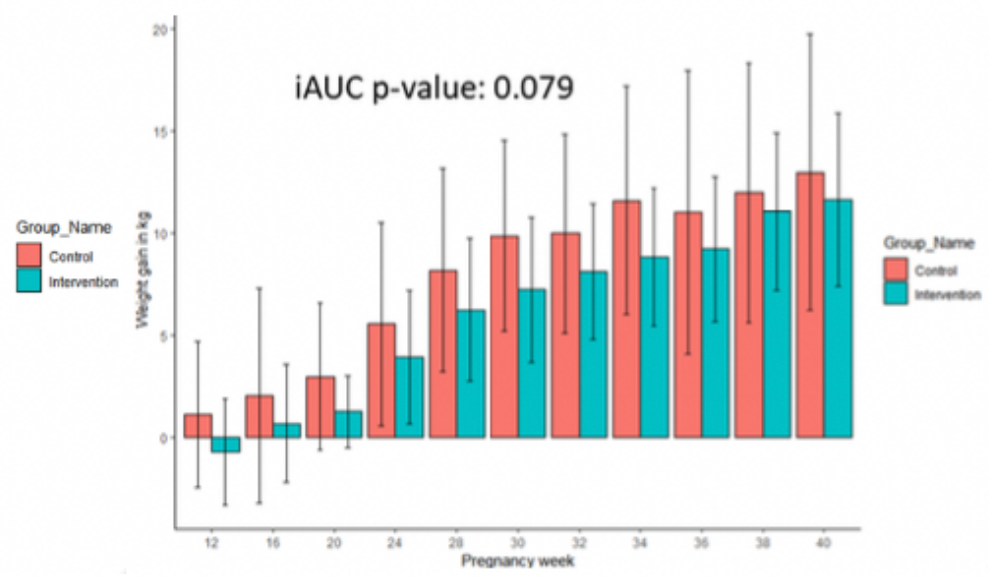

\section{Figure 4}

Weight gain by the subjects over the course of pregnancy (a: all eligible subjects; b: overweight/obese subjects; shown as mean \pm SD)

a.

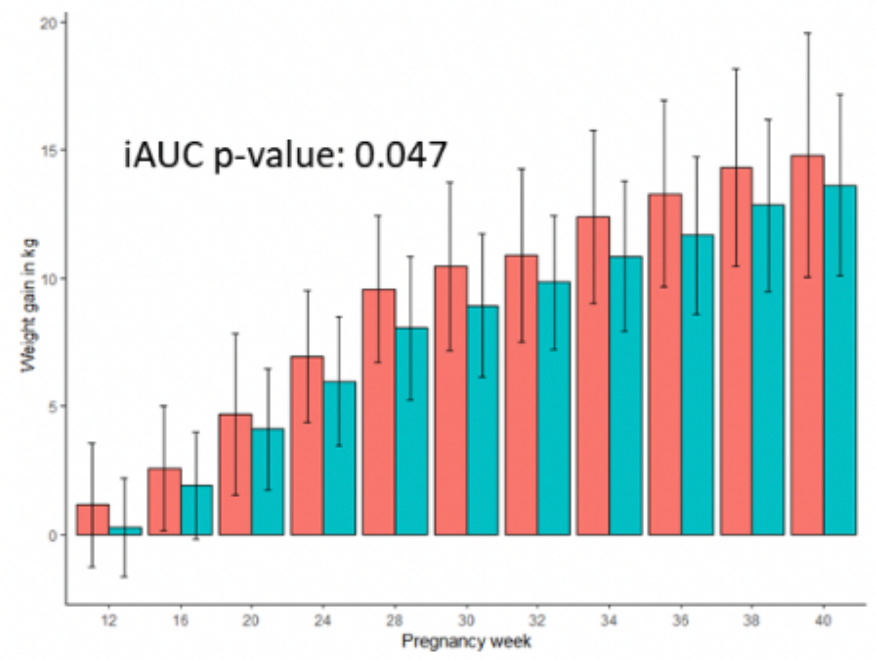

b.

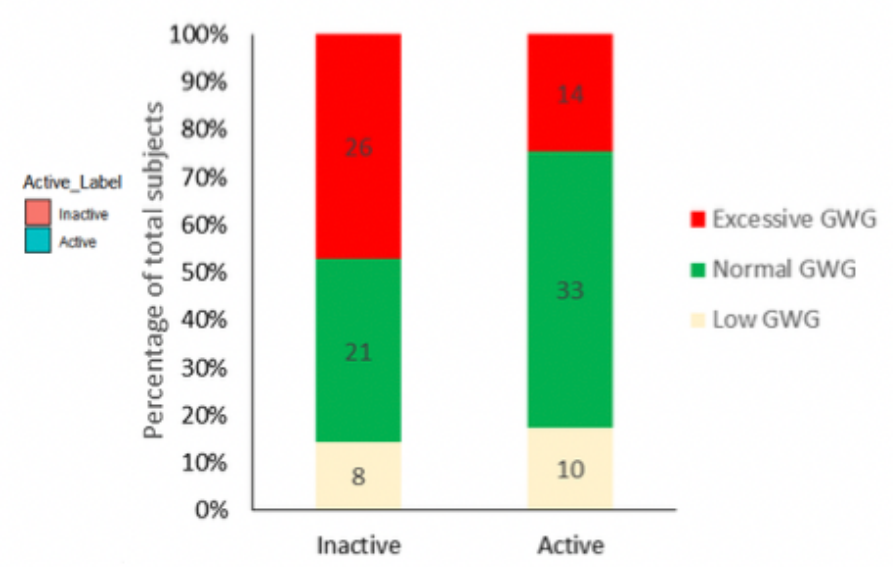

\section{Figure 5}

Weight gain for active and inactive Pregnancy Butler users (a: weight gain over the course of pregnancy, shown as mean \pm SD; b: breakdown of final GWG by IOM classifications, subject number and percentage) 
a.

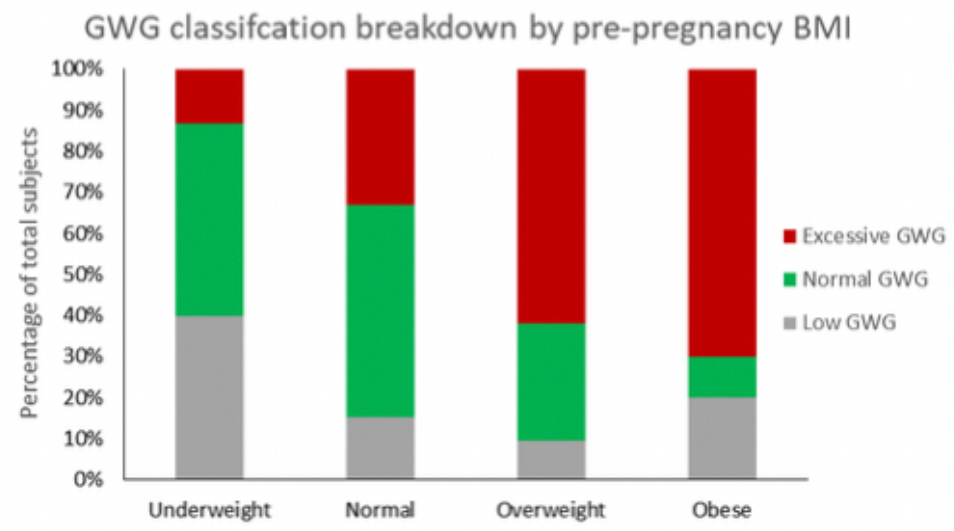

b.

GDM incidence vs. pre-determined risk

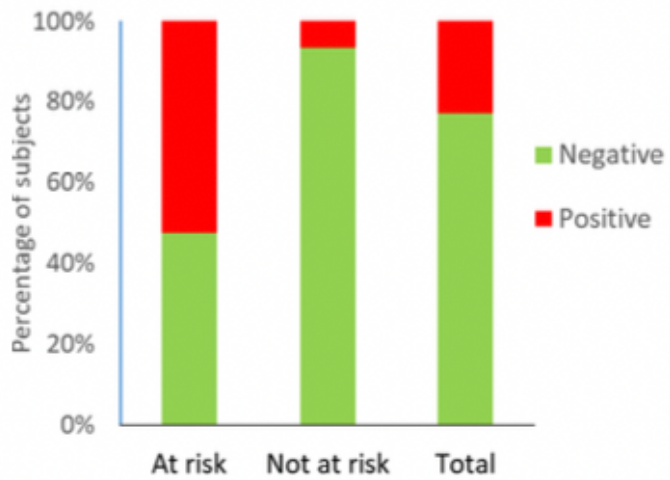

\section{Figure 6}

Predictors for eGWG and GDM risks (a: the incidence of gaining excessive weight at delivery for subjects with different pre-pregnancy BMl; $b$ :the incidence of diagnosed with GDM for subjects determined at-risk) 